\title{
Intrarectal administration of milk fat globule epidermal growth factor-8 protein ameliorates murine experimental colitis
}

\author{
AYA OTANI $^{1}$, SHUNJI ISHIHARA ${ }^{1}$, Md MONOWAR AZIZ $^{2,3}$, NAOKI OSHIMA $^{1}$, \\ YOSHIYUKI MISHIMA ${ }^{1}$, ICHIRO MORIYAMA ${ }^{1}$, TAKAFUMI YUKI ${ }^{4}$, YUJI AMANO ${ }^{4}$, \\ Md MESBAHM UDDIN ANSARY ${ }^{1}$, and YOSHIKAZU KINOSHITA ${ }^{1}$
}

\author{
${ }^{1}$ Department of Internal Medicine II, Shimane University School of Medicine, Shimane, Japan; ${ }^{2}$ Center for Immunology \\ and Inflammation, The Feinstein Institute for Medical Research and ${ }^{3}$ Department of Surgical Research, \\ North Shore University Hospital and Long Island Jewish Medical Center, Manhasset, NY, USA; \\ ${ }^{4}$ Division of Gastrointestinal Endoscopy, Shimane University Hospital, Shimane, Japan
}

Received October 1, 2011; Accepted November 10, 2011

DOI: $10.3892 / \mathrm{ijmm} .2011 .866$

\begin{abstract}
Milk fat globule epidermal growth factor-8 (MFG-E8) promotes phagocytic clearance of apoptotic cells to maintain normal tissue homeostasis. However, its functions in intestinal inflammatory disorders are unknown. Since the pathogenesis of those disorders are due to abnormal interactions between intestinal epithelial cells (IECs) and microbial pathogens, we analyzed the effects of MFG-E8 on IECs to determine its protective role in murine experimental colitis. Expression of $\alpha_{v} \beta_{3}$-integrin in Colon-26 cells was examined by RT-PCR and immunostaining. Colon-26 cells were pretreated with recombinant wild-type and mutant MFG-E8 proteins, following stimulation with flagellin as an inducer of innate immunity, and the effects of the recombinant proteins on inhibition of nuclear factor $-\kappa B(N F-\kappa B)$ and inflammatory cytokine production in flagellin-stimulated Colon-26 cells were determined using a luciferase assay and EIA, respectively. Experimental colitis was induced in mice by intrarectal administration of trinitrobenzene sulfonic acid (TNBS). Recombinant proteins were then intrarectally administered into TNBS-induced colitic mice, after which disease activity parameters (body weight, colon length, histological score), as well as interleukin (IL)-6 and MIP-2 levels were determined in inflamed tissues. Flagellin-induced inflammatory cytokine production in vitro was significantly downregulated via $\alpha_{v} \beta_{3}$-integrin following pretreatment with wild-type MFG-E8 due to inhibition of $\mathrm{NF}-\kappa \mathrm{B}$ activation. In vivo, intrarectal treatment with wild-type MFG-E8, but not its mutant counterpart, significantly inhibited body weight loss, colon shortening and
\end{abstract}

Correspondence to: Dr Shunji Ishihara, Department of Internal Medicine II, Shimane Medical University, 89-1 Enya-cho, Izumo, Shimane, Japan

E-mail: si360405@med.shimane-u.ac.jp

Key words: milk fat globule epidermal growth factor- $8, \alpha_{v} \beta_{3^{-}}$ integrin, intestinal inflammation, apoptosis, nuclear factor- $\mathrm{\kappa} B$ histological inflammation induced by TNBS administration. Our findings suggest that MFG-E8 has anti-inflammatory effects on flagellin-induced inflamed intestinal epithelial cells and may be a useful therapeutic agent for colitis.

\section{Introduction}

The gut is a multi-layered organ in which various cell types contribute to maintain homeostasis mechanisms. Among those cell types, epithelial cells form a monolayer on the luminal side of the intestine and function as the first line of defense against microbial pathogens (1-3). Intestinal epithelial cells (IECs) can sense a variety of pathogen-associated molecular patterns (PAMPs) via a limited number of pattern recognition receptors (PRRs) and preserve the gut under physiological conditions (4-8). On the other hand, prolonged and uncontrolled immune activation via PRRs in IECs induce several gut immune-mediated disorders including inflammatory bowel disease (IBD) (9-13). Experimental approaches regarding the regulation of innate immune function in IECs may lead to the development of novel therapeutic options for IBD.

Milk fat globule-epidermal growth factor 8 (MFG-E8), a glycoprotein secreted by mammary epithelial cells, activated macrophages, and immature dendritic cells (DCs), enhances engulfment of dying cells by forming a bridge between phosphatidylserine (PS) on apoptotic cells and $\alpha_{\mathrm{v}} \beta_{3}$-integrin on phagocytes (14-16). This scavenging function avoids the release of numerous inflammatory mediators from apoptotic cells and physiologically contributes to maintaining the host immune system. Inefficient clearance of apoptotic cells in MFG-E8 null mice induces severe inflammation and immune responses due to infiltration of apoptotic cells in various organs (14-17). Several studies have revealed that MFG-E8 plays important roles in the pathogenesis of inflammatory and autoimmune diseases, as well as sepsis, atherosclerosis, and Alzheimer's disease (18-24).

MFG-E8-mediated therapeutic benefits have also been shown in various inflammatory disease models. Those effects are dependent not only on enhanced clearance of apoptotic 
cells, but also on other anti-inflammatory mechanisms (25-27). We recently reported that the recombinant MFG-E8 protein inhibits production of inflammatory cytokines in LPS-treated macrophages, which contributed to attenuating intestinal inflammation in murine experimental colitis by modulating $\alpha_{v} \beta_{3}$-integrin signaling (28). Although MFG-E8 is expressed in a wide variety of cell types to perform several cellular events and modulates immune responses in numerous conditions (25-27), its role and underlying mechanisms have been evaluated mainly in experiments that focused on non-epithelial cells such as macrophages. Since the crucial pathogenesis of colitis is due to abnormal interactions between IECs and microbial pathogens, we speculated that MFG-E8 may directly regulate IECs during an innate immune activated condition. In the present study, we aimed to examine the effects of MFG-E8 on IECs to determine whether it has a protective role in murine experimental colitis.

\section{Materials and methods}

Reagents, vectors, and antibodies. Trinitrobenzene sulfonic acid (TNBS; Sigma, St. Louis, MO, USA), Lipofectamine 2000 (Invitrogen, Carlsbad, CA, USA), purified flagellin from S. typhimurium (Invivogen), $\mathrm{pNF}-\kappa \mathrm{B}-\mathrm{Luc}$ (Stratagene, La Jolla, CA, USA), pRL-TK (Promega, Madison, WI, USA), ELISA kits for interleukin (IL)-6, and CXCL2/macrophage inflammatory protein (MIP)-2 (R\&D Systems, Minneapolis, MN, USA) were acquired from their respective suppliers. The antibodies used were anti-mouse MFG-E8 (MBL, Nagoya, Japan), PE-anti- $\alpha_{\mathrm{v}}$-integrin, and FITC-anti- $\beta_{3}$-integrin, (eBioscience, San Diego, CA, USA), and were obtained from their respective manufacturers.

Cell culture. The mouse colon cancer cell line Colon-26 was obtained from the American Type Culture Collection (ATCC, Manassas, VA, USA) and grown in RPMI-1640 medium (Invitrogen), supplemented with $10 \%$ fetal bovine serum (FBS) (ICN Biomedicals, Inc., Aurora, OH, USA) and penicillinstreptomycin-amphotericin B (Invitrogen), and maintained at $37^{\circ} \mathrm{C}$ in $5 \% \mathrm{CO}_{2}$ in a humidified incubator.

Immunofluorescence protocol. Colon-26 cells $\left(1 \times 10^{5}\right.$ cells $\left./ \mathrm{ml}\right)$ were grown on glass chamber slides (BD Biosciences, Japan), then fixed in $4 \%$ paraformaldehyde and allowed to react with PE-labeled anti-mouse $\alpha_{v}$ and FITC-labeled $\beta_{3}$-integrin antibodies for $60 \mathrm{~min}$ at room temperature. After subsequent washing with cold PBS, $\alpha_{v} \beta_{3}$-integrin expression in Colon-26 cells was examined using an Olympus BX51 fluorescent microscope (Olympus, Japan).

Recombinant MFG-E8proteins. We prepared mouse wild-type and RGD mutant MFG-E8 proteins using mammalian expression systems, as described previously (28). The functional efficiency of both proteins has been confirmed in our recent studies (28). The RGD domain is a key part of the MFG-E8 protein and can bind to $\alpha_{\mathrm{v}} \beta_{3}$-integrin on apoptotic cells. The RGD mutant MFG-E8 (D89E) was previously shown to be a dominant negative form of MFG-E8 $(14,15)$.

Transfection and luciferase assay. For in vitro transfection experiments, Colon-26 cells were grown and plated on 24-well plates $\left(2.5 \times 10^{4}\right.$ cells/well $)$ in RPMI-1640 with $10 \%$ FBS in a humidified chamber. After 18-24 h, the cells reached $50 \%$

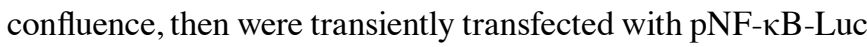
(200 ng/well) and pRL-TK-Renilla-Luc (20 ng/well) using Lipofectamine 2000 (2.5 $\mu \mathrm{l} /$ well), after which nuclear factor- $\kappa \mathrm{B}$ $(\mathrm{NF}-\kappa \mathrm{B})$ activity was measured using a dual-luciferase reporter assay system (Promega).

Colitis induction and treatment of recombinant $M F G-E 8$ proteins in mice. Seven-week-old male specific pathogen-free $\mathrm{BALB} / \mathrm{c}$ mice (Charles River, Japan) were housed according to our institutional guidelines following the approval of the Ethics Committee of Shimane University. For a TNBS-induced colitis model, mice were anesthetized for 90-120 min with an intraperitoneal pentobarbital injection and then given an intrarectal administration of TNBS $(100 \mu 1,125 \mathrm{mg} / \mathrm{kg})$ dissolved in $50 \%$ ethanol. Control mice received only $50 \%$ ethanol using the same technique. The in vivo study protocol is shown in Fig. 2B. Recombinant MFG-E8 proteins were diluted in PBS and $10 \mu \mathrm{l}$ of the solution $(1,200 \mathrm{ng} /$ body) was intrarectally injected before TNBS administration on Day 0 . The body weight (BW) of each mouse was measured daily until euthanasia. At the end of TNBS-induced colitis, the mice were euthanized, and their colonic tissues were dissected for histology and EIA.

Histological examination. For histology, 3- $\mu \mathrm{m}$ formalin-fixed paraffin-embedded colon tissue sections were stained with hematoxylin and eosin (H\&E), then examined under a light microscope by two investigators in a double-blinded fashion. Histological grading was evaluated as previously described $(29,30)$. Three different parameters were considered, severity of inflammation (based on polymorphonuclear neutrophil infiltration; 0-3: none, slight, moderate and severe), depth of injury (0-3: none, mucosal, mucosal and submucosal, transmural), and crypt damage (0-4: none, basal one-third damaged, basal two-thirds damaged, only surface epithelium intact, entire crypt and epithelium lost). The score for each parameter was multiplied by a factor reflecting the percentage of tissue involvement (x1, 0-25\%; x2, 26-50\%; x3, 51-75\%; $\mathrm{x} 4,76-100 \%)$, then all values were added to a sum with a maximum possible score of 40 .

$R T$ and quantitative real-time PCR. Total-RNA was extracted from colonic tissues and cultured cells using Isogen (Nippon Gene, Japan) and then equal amounts of DNase I (Ambion, Inc., Austin, TX, USA) treated RNA were reverse transcribed into cDNA using a QPCR cDNA kit (Stratagene). A real-time fluorescence PCR assay based on SYBR-Green (Applied Biosystems, Warrington, UK) was then performed using the following primers: MFG-E8, forward, 5'-CGGGCCAAGACAATGAC ATC-3' and reverse, 5'-TCTCTCAGTCTCATTGCACAC AAG-3'; $\beta$-actin, forward, 5'-CGTGAAAAGATGACCCAGA TCA-3' and reverse, 5'-CACAGCCTGGATGGCTACGTA-3'. To screen $\alpha_{v}$ - and $\beta_{3}$-integrin gene expressions in Colon-26 cells, reverse transcriptase PCR (RT-PCR) was performed using the following primers: $\alpha_{\mathrm{v}}$-integrin, forward, 5'-CTGGC TGTGTTGGTATTTGTAATGTAC-3' and reverse, 5'-AGC TGTTCTCGTTCTTGCTCTTC-3'); $\beta_{3}$-integrin, forward, 5'-CGTCAGCCTTTACCAGAATTATAGTG-3' and reverse, 5'-TTTCCCGTAAGCATCAACAATG-3'). 
A
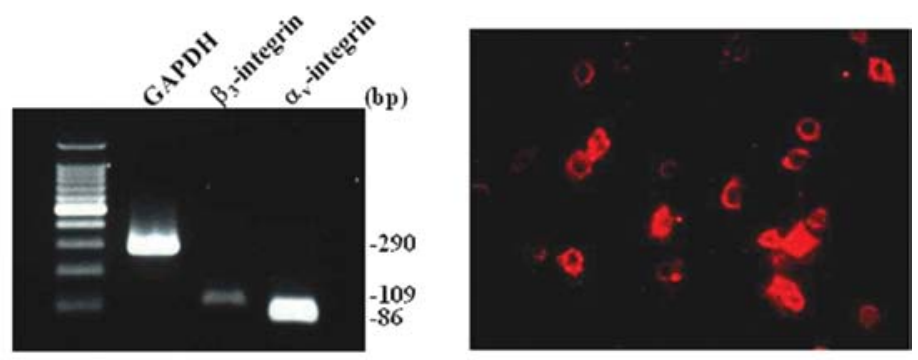

$\alpha_{v}$-integrin



$\beta_{3}$-integrin

B
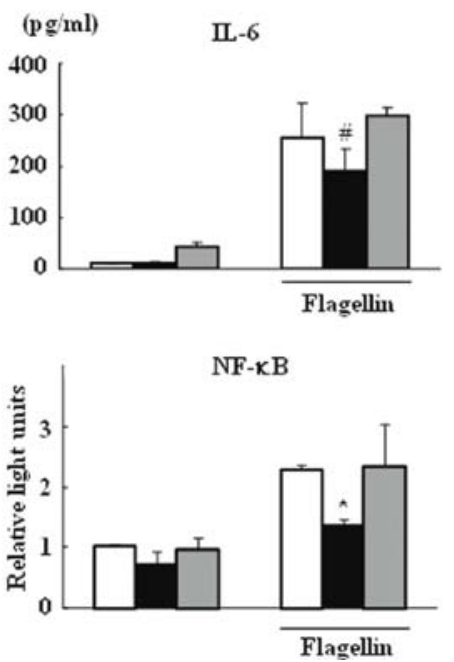

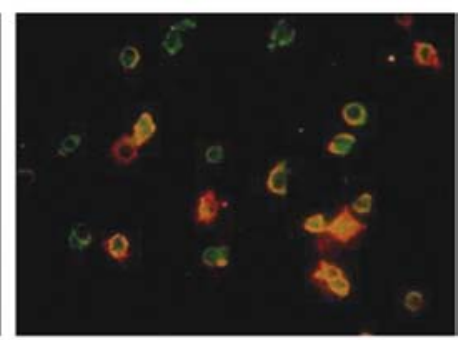

Merge
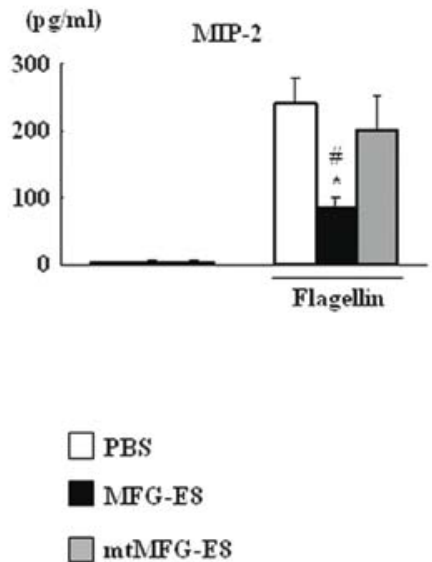

Figure 1. (A) Expressions of $\alpha_{\mathrm{v}}$ - and $\beta_{3}$-integrins in Colon-26 cells. The gene expressions of $\alpha \mathrm{v}$ - and $\beta 3$-integrins were examined using RT-PCR (upper left panel). Protein expression and cellular localization of $\alpha_{\mathrm{v}}$ - and $\beta_{3}$-integrins were detected by immunofluorescence staining using PE-labeled anti-mouse $\alpha_{v}$ and FITC-labeled $\beta_{3}$-integrin antibodies. (B) Effects of recombinant wild-type and mutant (mt) MFG-E8 proteins on inflammatory cytokine production in flagellin-stimulated Colon-26 cells. Twenty-four hours after stimulation with or without flagellin and recombinant MFG-E8 proteins, the culture supernatants were used for measurements of IL- 6 and MIP-2 by EIA. Error bars indicate the SEM values obtained from three independent experiments. ${ }^{*}<0.05$ vs. flagellin with PBS; ${ }^{\text {P }}<0.05$ vs. flagellin with mtMFG-E8.

Enzyme immune assay (EIA). Mouse IL-6 and MIP-2 contents in colonic tissues and culture supernatants were determined using EIA according to the manufacturer's protocol. Briefly, total proteins from distal colonic tissues were extracted using lysis buffer (200 mM NaCl, $5 \mathrm{mM}$ EDTA, $10 \mathrm{mM}$ Tris, $10 \%$ glycine, and $1 \mathrm{mM}$ PMSF, pH 7.4) and subjected to EIA using mouse IL-6 and MIP-2 EIA kits. In vitro culture supernatants from Colon-26 cells treated with or without flagellin and recombinant MFG-E8 proteins were also examined for IL-6 and MIP-2 contents by EIA, as described above.

Statistical analysis. All data are expressed as the mean \pm standard error of the mean (SEM). The Student's t-test was used for statistical determinations. P-values $<0.05$ were considered to be significant.

\section{Results}

Expressions of $\alpha_{v}$-and $\beta_{3}$-integrins in Colon-26 cells. Although the $\alpha_{v} \beta_{3}$-integrin complex is essential for MFG-E8-mediated cellular functions in targeted cells, its expression and function remains largely unknown. Initially, we examined the gene expressions of $\alpha_{v}$ - and $\beta_{3}$-integrins in Colon-26 cells (mice colonic epithelial cell line) using RT-PCR. Electrophoresis results clearly showed the expressions of both in Colon-26 cells (Fig. 1A). In addition, immunofluorescence staining was performed to evaluate the protein levels of $\alpha_{\mathrm{v}}$ - and $\beta_{3}$-integrins and their cellular localization (Fig. 1A). Abundant immunoreactive signals for $\alpha_{v^{-}}$(red) and $\beta_{3^{-}}$(green) integrins were observed on the cellular surface, and both integrins merged within the same cells. 
A

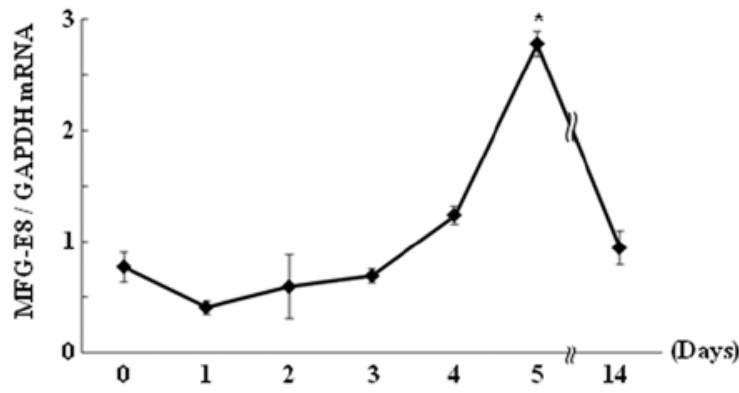

$\mathrm{C}$

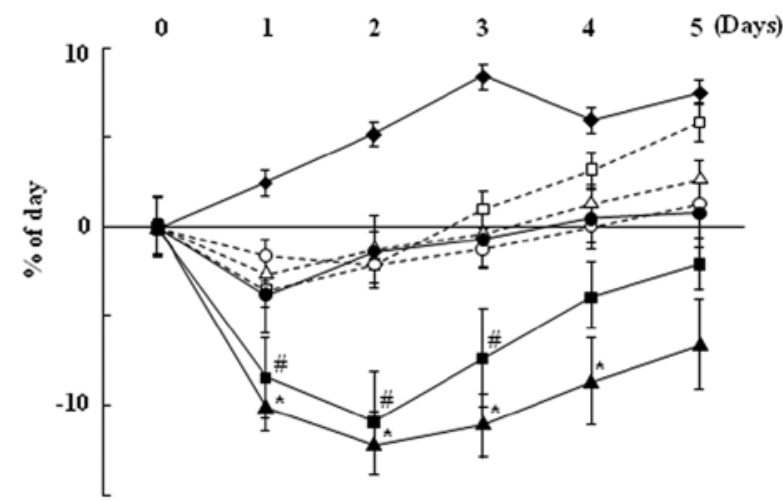

B

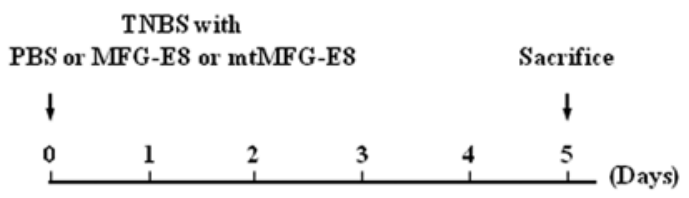

Figure 2. (A) Time course changes of MFG-E8 mRNA expression in inflamed colons during TNBS-induced colitis (n=4). Error bars indicate the standard error of mean values. ${ }^{*} \mathrm{P}<0.05$ vs. Day 0. (B) Protocol for experimental colitis and treatment with recombinant MFG-E8 proteins. (C) Effects of recombinant wild-type and mutant (mt) MFG-E8 proteins on body weight changes in TNBS-treated mice (control and non-TNBS groups, 5 mice/group; TNBS-treated groups, 9-14 mice/group). Data are expressed as serial changes in the percentage of weight change during TNBS-induced colitis. * $<<0.05$ vs. TNBS-treated with PBS; ${ }^{\text {} P}<0.05$ vs. TNBS-treated with mtMFG-E8.

$M F G-E 8$ inhibits production of inflammatory cytokines in flagellin-stimulated Colon-26 cells via $\alpha_{v} \beta_{3}$-integrin in an $N F-\kappa B$-dependent manner. After observing the expression of $\alpha_{\mathrm{v}} \beta_{3}$-integrin in IECs, we next examined the direct effects of MFG-E8 on production of inflammatory cytokines in IECs. Flagellin is a specific microbial ligand of TLR5 and has been shown to be a potent stimulator of inflammatory cytokines in IECs. Thus, we used it as a ligand for induction of inflammatory cytokines in cultured Colon-26 cells. Twenty-four hours after stimulation with or without flagellin and recombinant MFG-E8 proteins, culture supernatants were subjected to measurements of IL- 6 and MIP- 2 by EIA. Flagellin markedly induced IL- 6 and MIP-2 in the culture supernatants, while cells treated with wildtype MFG-E8 protein showed significantly decreased effects of flagellin on cytokine production. Mutant MFG-E8 protein had no effects on flagellin-stimulated Colon-26 cells (Fig. 1B). $\mathrm{NF}-\mathrm{\kappa B}$ is a potent transcription factor that regulates the expressions of various inflammatory genes, including IL-6 and MIP-2 . We also examined the effects of wild-type and mutant MFG-E8 proteins on NF- $\mathrm{kB}$ activity in flagellin-stimulated Colon-26 cells. Following flagellin stimulation for $12 \mathrm{~h}, \mathrm{NF}-\mathrm{\kappa B}$ activity was assessed using a dual-luciferase assay system. Cells treated with wild-type MFG-E8 showed significant downregulation of flagellin-induced NF- $\mathrm{kB}$ activity, whereas mutant MFG-E8 had no significant effects on that activity (Fig. 1B).

Intrarectal administration of $M F G-E 8$ protects mice from TNBS-induced colitis. Since MFG-E8 inhibited inflammatory cytokine production in flagellin-stimulated IECs, we speculated that intrarectal administration of recombinant wild-type MFG-E8 protein may ameliorate intestinal inflammation by protecting IECs from stimulation by luminal pathogen-associated molecules. Before carrying out our in vivo examination, we evaluated the time course changes of MFG-E8 expression during TNBS-induced colitis. The MFG-E8 was downregulated in inflamed colons during the acute and severe inflammatory phases of colitis (Days 1 to 3), while it become upregulated during the healing phase (Days 4 to 5) (Fig. 2A).

Based on results showing the altered expression of MFG-E8 during TNBS-induced colitis, we designed an in vivo study protocol (Fig. 2B). To assess the direct effects of MFG-E8 on IECs, recombinant wild-type and mutant MFG-E8 proteins were intrarectally injected into mice before TNBS administration, while other mice were injected with PBS instead of MFG-E8 proteins. Mice without TNBS administration were also assessed as controls. In mice who received TNBS with PBS or mutant MFG-E8 protein, body weight loss was clearly observed, while those injected with wild-type MFG-E8 showed significantly lower body weight loss from Days 1 to 3 (Fig. 2C). In mice not treated with TNBS, injection of recombinant proteins had no influence on body weight changes.

Representative specimens dissected from each experimental group are shown in Fig. 3A. Colons dissected from the wild-type MFG-E8-treated group showed increased length and decreased weight as compared to those from the PBS- and mutant MFG-E8-treated TNBS groups (Fig. 3). Histological 
A

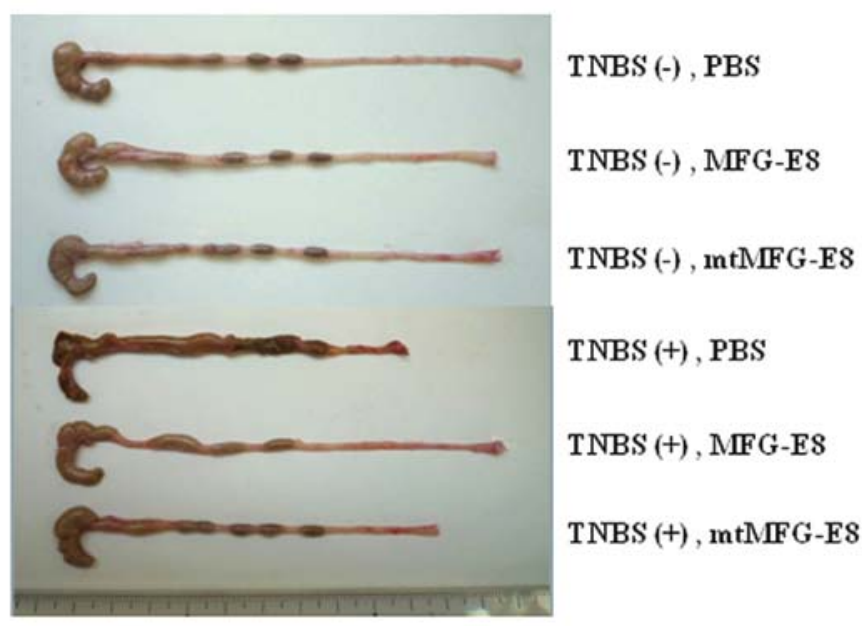

B

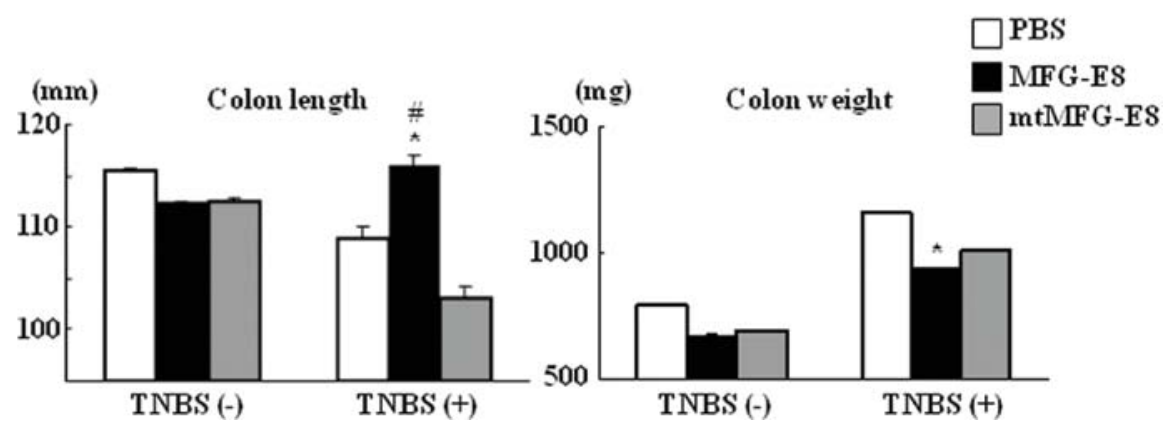

Figure 3. (A) Representative images of colons dissected from experimental mice. (B) Effects of recombinant wild-type and mutant (mt) MFG-E8 proteins on colon length and weight in mice with or without TNBS treatment (control and non-TNBS groups, 5 mice/group; TNBS-treated groups, 9-14 mice/group). Error bars indicate the SEM values. "P<0.05 vs. TNBS-treated with PBS; ${ }^{\#} \mathrm{P}<0.05$ vs. TNBS-treated with mtMFG-E8.

examinations also showed that inflammation, extent of injury, and crypt damage were significantly decreased in the wildtype MFG-E8-treated colitic mice (Fig. 4). Total histological scores in the dissected colon samples from the wild-type MFG-E8-treated colitic mice were also markedly lower than in those from the other groups (Fig. 4B). Furthermore, to evaluate the effects of MFG-E8 on inflammatory cytokine production, protein was extracted from colonic tissues, and IL-6 and MIP-2 contents were measured. Treatment with wildtype MFG-E8 protein significantly downregulated the tissue contents of IL- 6 and MIP-2 as compared to those in PBS- and mutant MFG-E8 protein-treated colitic mice (Fig. 5).

\section{Discussion}

The present findings revealed that MFG-E8 inhibits flagellinmediated production of inflammatory cytokines in cultured IECs by modulating NF- $\mathrm{KB}$ activation via $\alpha_{\mathrm{v}} \beta_{3}$-integrin. In addition, our results demonstrated that intrarectal administration of recombinant wild-type MFG-E8 protein ameliorates TNBS-induced mice colitis. These are the first known results to show an anti-inflammatory role of MFG-E8 in IECs and its therapeutic potential for intestinal inflammation.

MFG-E8 was originally described as a factor for efficient phagocytosis of apoptotic cells for maintaining immune homeostasis $(14,15)$. Apart from this conventional role, diverse functions of MFG-E8 have also been seen in several cellular and immune-mediated disorders (18-24). Recent reports revealed the importance of this secreted protein on regulation of the innate immune system, as well as an anti-inflammatory role and its participation in regeneration of injured tissues $(25-27)$. We previously reported that the anti-inflammatory role of recombinant MFG-E8 protein in dextran sulphate sodium (DSS)-induced colitis was exerted by modulating LPS-induced innate-immune responses in macrophages (28). A recent study also revealed that DSS-induced colonic inflammation in MFG-E8 knockout mice was more severe than that in wild-type mice (31). Moreover, the anti-inflammatory effect of the recombinant MFG-E8 protein was shown in a mouse model of ischemia and reperfusion-induced intestinal injuries (23). Thus, the beneficial effect of MFG-E8 on downregulation of inflammation is recognized. However, the protective role of MFG-E8 in intestinal injuries in those studies was only noted in the context of non-epithelial mononuclear cells, and they did not investigate the interaction between MFG-E8 and IECs.

Considering the novel function of MFG-E8 to directly attenuate intestinal inflammation, we utilized colonic epithelial cells because of their pivotal role in the first line of defense against bacterial pathogens during intestinal inflammation. We previously reported differential expression of TLRs in IECs during intestinal inflammation in a mouse model of colitis (5), which was shown to contribute to regulating gut immunity, barrier function, and regeneration of injured tissues in various studies. Among the various kinds of TLR ligands, flagellin is a specific microbial ligand of TLR5, which is a pathogenic factor that induces a variety of inflammatory disorders including 
A

PBS



B
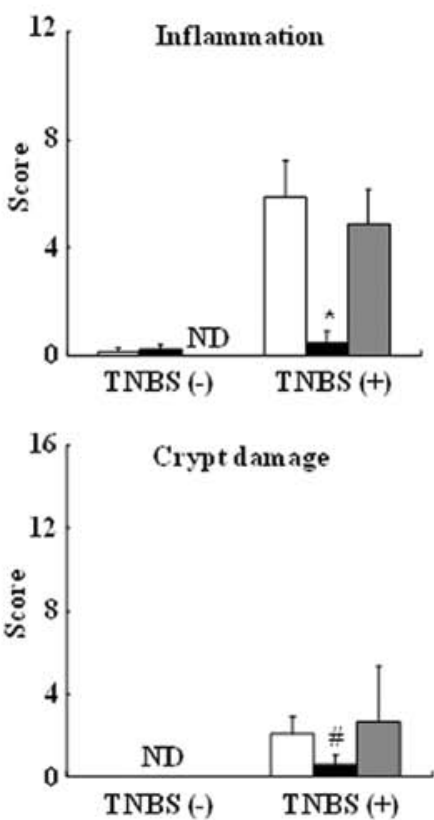

MFG-E8
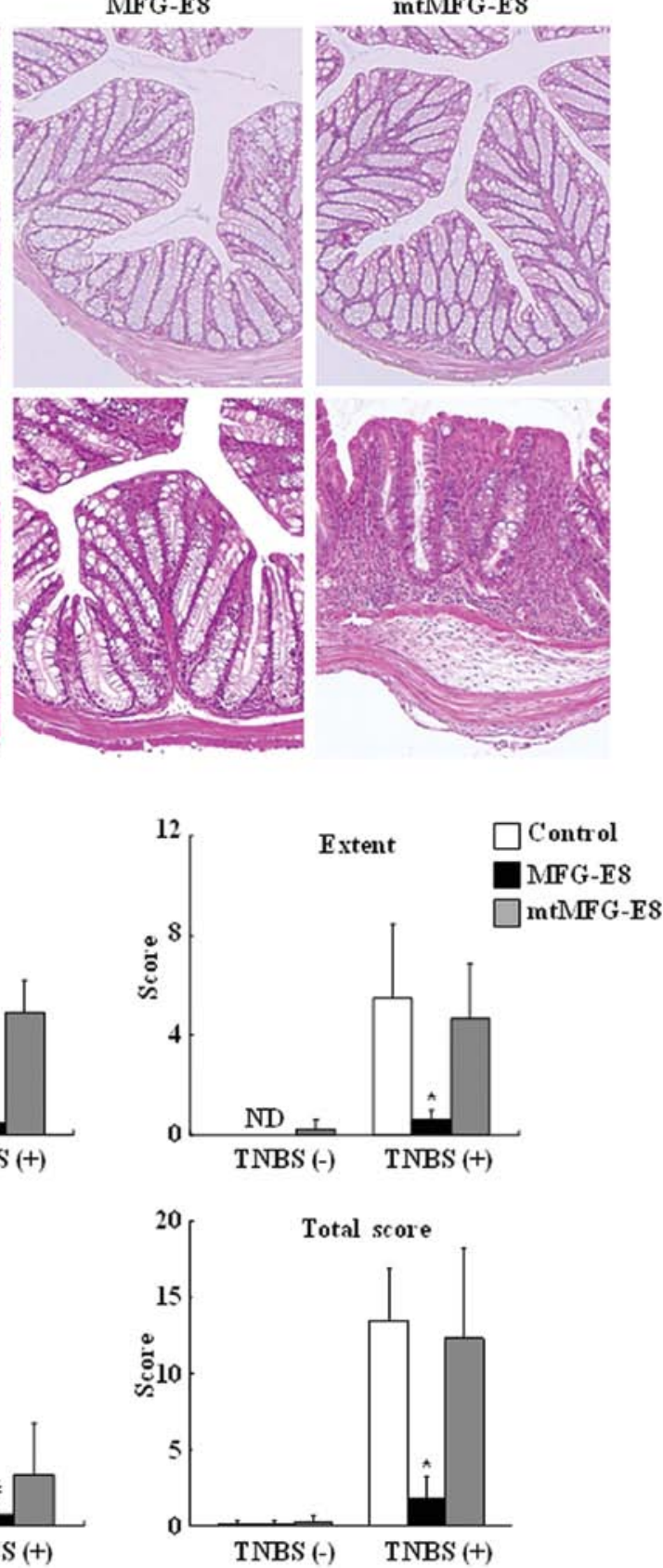

Figure 4. (A) Representative histological images of colonic samples dissected from experimental mice. (B) Effects of recombinant wild-type and mutant (mt) MFG-E8 proteins on histological scores in mice with or without TNBS treatment. Error bars indicate the SEM values obtained independently from 5 mice. ${ }^{*} \mathrm{P}<0.05$ vs. TNBS-treated with $\mathrm{PBS} ;{ }^{*} \mathrm{P}<0.05$ vs. TNBS-treated with mtMFG-E8.

IL-6

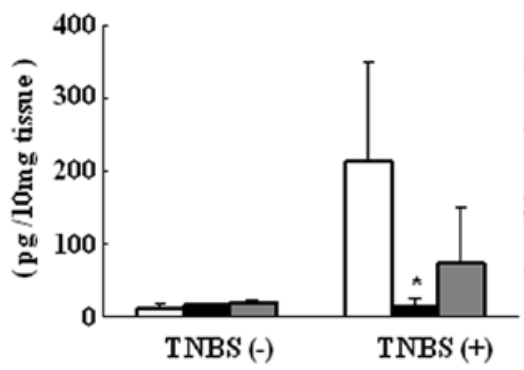

MIIP-2

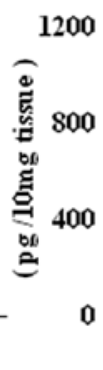

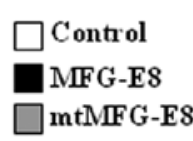

Figure 5. Effects of recombinant wild-type and mutant (mt) MFG-E8 proteins on IL-6 and MIP-2 in mice with or without TNBS treatment. Total protein was extracted from each distal colonic tissue sample. Cytokine contents were evaluated using EIA. Error bars indicate the SEM values obtained independently from 5 mice. ${ }^{*} \mathrm{P}<0.05$ vs. TNBS-treated with PBS; ${ }^{\mathrm{P}}<0.05$ vs. TNBS-treated with mtMFG-E8. 
infectious enteritis and IBD (32). We recently demonstrated that flagellin can stimulate abundant production of IL-8 in cultured IECs $(8,33)$. Based on our previous findings, we used flagellin for the present in vitro experiments to clarify the antiinflammatory role of MFG-E8 in IECs.

We initially examined $\alpha_{v} \beta_{3}$-integrin expression in mice colonic epithelial cells (Colon-26), and found its significant expression on the cellular surface. Other in vitro studies have also shown that recombinant wild-type MFG-E8 protein significantly inhibits the production of IL- 6 and MIP- 2 by decreasing NF- $\mathrm{kB}$ activation in flagellin-stimulated Colon-26 cells. In contrast, no protective effect was not found in mutant proteintreated cells. These results suggest that the anti-inflammatory function of MFG-E8 is dependent on $\alpha_{v} \beta_{3}$-integrin expression in IECs, because the mutant protein targeting the RGD domain of MFG-E8 decreases its binding to $\alpha_{v} \beta_{3}$-integrin on the cells.

Among the various physiological conditions in the gut, tolerant conditions are essentially preserved between a monolayer of IECs and various luminal microbial pathogens by modulating appropriate innate immune levels (1-3). However, when intestinal injuries occur, numerous luminal microbial components such as flagellin abnormally contact with IECs and various mucosal cells (32). Notably, innate immune response to pathogens is rapidly induced in IECs during the early phase of intestinal inflammation. In the present study, we focused on this point and injected intrarectally recombinant MFG-E8 proteins into mice before treatment with TNBS. We found that robust induction of IL- 6 and MIP-2 during the acute phase of TNBSinduced colitis was significantly downregulated in the presence of wild-type MFG-E8 protein. As observed in colonic histology findings, comparatively fewer infiltrating mononuclear cells were present in the lamina propria zone, due to the reduced production of IL-6 and MIP-2 in inflamed mucosa of wild-type MFG-E8-treated mice. On the other hand, such a protective role was not seen in mice treated with mutant MFG-E8 protein. These results indicate a therapeutic benefit of intrarectal administration of wild-type MFG-E8 protein for intestinal inflammation. Since MFG-E8 can inhibit excess innate immune responses in various non-epithelial immune cells, such anti-inflammatory effects might also have contributed to the amelioration of TNBS colitis seen in the present study.

Recently, Bu et al (34) established an in vitro woundhealing model to evaluate the effects of MFG-E8 on IEC migration. They observed that recombinant MFG-E8 protein bound to phosphatidylserine (PS) on IECs at the monolayer wound-edge in vitro and that binding stimulated IEC migration by activating intracellular protein kinase $\mathrm{C}$ (PKC). They also demonstrated that treatment of experimental septic mice with recombinant MFG-E8 accelerated mucosal healing by binding to PS on the injured IECs, suggesting that MFG-E8 plays a crucial role in tissue regeneration during the healing process of injured colonic mucosa. On the other hand, in the present study, we focused on $\alpha_{v} \beta_{3}$-integrin expression in IECs and confirmed its association with the anti-inflammatory role of MFG-E8. Thus, MFG-E8 has a variety of effects on IECs to modulate inflammation and regeneration in the intestines.

In conclusion, the present findings showed a beneficial anti-inflammatory role of intrarectal administration of recombinant MFG-E8 protein in a murine model of colitis. However, there are several issues that require further clarification before clinical use. The precise mechanisms related to downregulation of NF- $\kappa \mathrm{B}$ activity mediated by $\alpha_{\mathrm{v}} \beta_{3}$-integrin signaling should be elucidated. In addition, prior to administration of MFG-E8 for chronic, relapsing intestinal disorders such as IBD, its role in chronic models of colitis must be further addressed. Moreover, though we assessed the effects of recombinant MFG-E8 protein in non-colitic healthy mice, MFG-E8 was administered only for a short period and the long-term effects in regard to physiological, immunological, and clinical aspects must be evaluated in the future.

\section{Acknowledgements}

This study was supported in part by Health and Labour Sciences Research Grants for research of intractable diseases from the Ministry of Health, Labour and Welfare of Japan.

\section{References}

1. Cario E, Rosenberg IM, Brandwein SL, Beck PL, Reinecker HC and Podolsky DK: Lipopolysaccharide activates distinct signaling pathways in intestinal epithelial cell lines expressing Toll-like receptors. J Immunol 164: 966-972, 2000.

2. Monteleone I, Vavassori P, Biancone L, Monteleone G and Pallone F: Immunoregulation in the gut: success and failures in human disease. Gut 50 (Suppl 3): S60-S64, 2002.

3. Rakoff-Nahoum S, Paglino J, Eslami-Varzaneh F, Edberg S and Medzhitov R: Recognition of commensal microflora by toll-like receptors is required for intestinal homeostasis. Cell 118: 229-241, 2004.

4. Hausmann M, Kiessling S, Mestermann S, et al: Toll-like receptors 2 and 4 are upregulated during intestinal inflammation. Gastroenterology 122: 1987-2000, 2002.

5. Ortega-Cava CF, Ishihara S, Rumi MA, et al: Strategic compartmentalization of Toll-like receptor 4 in the mouse gut. J Immunol 170: 3977-3985, 2003.

6. Abreu MT, Fukata M and Arditi M: TLR signaling in the gut in health and disease. J Immunol 174: 4453-4460, 2005.

7. Kadota C, Ishihara S, Aziz MM, et al: Downregulation of single immunoglobulin interleukin-1R-related molecule (SIGIRR)/ TIR8 expression in intestinal epithelial cells during inflammation. Clin Exp Immunol 162: 348-361, 2010.

8. Li YY, Ishihara S, Aziz MM, et al: Autophagy is required for toll-like receptor-mediated interleukin-8 production in intestinal epithelial cells. Int J Mol Med 27: 337-344, 2011.

9. Akira S, Uematsu S and Takeuchi O: Pathogen recognition and innate immunity. Cell 124: 783-801, 2006.

10. Ishihara S, Aziz MM, Yuki T, Kazumori H and Kinoshita Y: Inflammatory bowel disease: review from the aspect of genetics. J Gastroenterol 44: 1097-1108, 2009.

11. Mayer L: Evolving paradigms in the pathogenesis of IBD. J Gastroenterol 45: 9-16, 2010.

12. Engel MA and Neurath MF: New pathophysiological insights and modern treatment of IBD. J Gastroenterol 45: 571-583, 2010.

13. Andoh A, Imaeda H, Aomatsu T, et al: Comparison of the fecal microbiota profiles between ulcerative colitis and Crohn's disease using terminal restriction fragment length polymorphism analysis. J Gastroenterol 46: 479-486, 2011.

14. Hanayama R, Tanaka M, Miwa K, Shinohara A, Iwamatsu A and Nagata S: Identification of a factor that links apoptotic cells to phagocytes. Nature 417: 182-187, 2002.

15. Hanayama R, Tanaka M, Miyasaka K, Aozasa K, Koike M, Uchiyama $Y$ and Nagata S: Autoimmune disease and impaired uptake of apoptotic cells in MFG-E8-deficient mice. Science 304: 1147-1150, 2004.

16. Miyasaka K, Hanayama R, Tanaka M and Nagata S: Expression of milk fat globule epidermal growth factor 8 in immature dendritic cells for engulfment of apoptotic cells. Eur J Immunol 34: 1414-1422, 2004.

17. Peng Y and Elkon KB: Autoimmunity in MFG-E8-deficient mice is associated with altered trafficking and enhanced cross-presentation of apoptotic cell antigens. J Clin Invest 121: 2221-2241, 2011. 
18. Leonardi-Essmann F, Emig M, Kitamura Y, Spanagel R and Gebicke-Haerter PJ: Fractalkine-upregulated milk-fat globule EGF factor-8 protein in cultured rat microglia. J Neuroimmunol 160 92-101, 2005

19. Miksa M, Amin D, Wu R, Jacob A, Zhou M, Dong W, Yang WL, Ravikumar TS and Wang P: Maturation-induced downregulation of MFG-E8 impairs apoptotic cell clearance and enhances endotoxin response. Int J Mol Med 22: 743-748, 2007.

20. Jinushi M, Nakazaki Y, Dougan M, Carrasco DR, Mihm M and Dranoff G: MFG-E8-mediated uptake of apoptotic cells by APCs links the pro- and antiinflammatory activities of GM-CSF. J Clin Invest 117: 1902-1913, 2007.

21. Komura H, Miksa M, Wu R, Goyert SM and Wang P: Milk fat globule epidermal growth factor-factor VIII is downregulated in sepsis via the lipopolysaccharide-CD14 pathway. J Immunol 182: 581-587, 2009.

22. Kranich J, Krautler NJ, Falsig J, et al: Engulfment of cerebral apoptotic bodies controls the course of prion disease in a mouse strain-dependent manner. J Exp Med 207: 2271-2281, 2010.

23. Cui T, Miksa M, Wu R, et al: Milk fat globule epidermal growth factor 8 attenuates acute lung injury in mice after intestinal ischemia and reperfusion. Am J Respir Crit Care Med 181: 238-246, 2010.

24. Kruse K, Janko C, Urbonaviciute V, et al: Inefficient clearance of dying cells in patients with SLE: anti-dsDNA autoantibodies, MFG-E8, HMGB-1 and other players. Apoptosis 15: 1098-1113, 2010.

25. Litvack ML and Palaniyar N: Review: soluble innate immune pattern-recognition proteins for clearing dying cells and cellular components: implications on exacerbating or resolving inflammation. Innate Immun 16: 191-200, 2010.

26. Aziz M, Jacob A, Matsuda A and Wang P: Review: milk fat globule-EGF factor 8 expression, function and plausible signal transduction in resolving inflammation. Apoptosis 16: 1077-1086, 2011.
27. Kusunoki R, Ishihara S, Aziz MM,Oka A, Tada Y and Kinoshita Y: Roles of milk fat globule-EGF factor 8 in intestinal inflammation. Digestion (In press).

28. Aziz MM, Ishihara S, Mishima Y, et al: MFG-E8 attenuates intestinal inflammation in murine experimental colitis by modulating osteopontin-dependent alphavbeta3 integrin signaling. J Immunol 182: 7222-7232, 2009.

29. Vowinkel T, Kalogeris TJ, Mori M, et al: Impact of dextran sulfate sodium load on the severity of inflammation in experimental colitis. Dig Dis Sci 49: 556-564, 2004.

30. Moriyama I, Ishihara S, Rumi MA, et al: Decoy oligodeoxynucleotide targeting activator protein-1 (AP-1) attenuates intestinal inflammation in murine experimental colitis. Lab Invest 88 : 652-663, 2008

31. Chogle A, Bu HF, Wang X, Brown JB, Chou PM and Tan XD: Milk fat globule-EGF factor 8 is a critical protein for healing of dextran sodium sulfate-induced acute colitis in mice. Mol Med 17: 502-507, 2011.

32. Rhee SH, Im E, Riegler M, Kokkotou E, O'brien M and Pothoulakis C: Pathophysiological role of Toll-like receptor 5 engagement by bacterial flagellin in colonic inflammation. Proc Natl Acad Sci USA 102: 13610-13615, 2005.

33. Oshima N, Ishihara S, Rumi MA, et al: A20 is an early responding negative regulator of Toll-like receptor 5 signalling in intestinal epithelial cells during inflammation. Clin Exp Immunol 159: 185-198, 2010.

34. Bu HF, Zuo XL, Wang X, et al: Milk fat globule-EGF factor 8/ lactadherin plays a crucial role in maintenance and repair of murine intestinal epithelium. J Clin Invest 117: 3673-3683, 2007. 